\title{
Ueber den Knabenüberschuss der älteren Erstgebärenden
}

nebst einem Beitrage zum Hofacker-Sadler'schen Gesetze. Von

Dr. F. Ahlfeld.

(Unter Benutzung einiger Resultate aus der Dissertation des Hern Dr. Schramm aus Braunschweig.)

In einer Arbeit über die Geburten älterer Erstgeschwängerten (dieses Archiv, Band IV, Seite 510) fand ich das auffallende Resultat, dass von 103 Früchten 59 männlichen, 43 weiblichen Geschlechtes waren. Von einer Frucht war das Geschlecht unbekannt. Statt des bekannten Verhältnisses von 106 Knaben zu 100 Mädchen erhielten wir hier ein Verhältniss von 137:100. Diese Zahlen gewannen an Bedeutung, als Hecker in einem Aufsatze, in dem er das gleiche Thema behandelt (dieses Archiv, Band VII, Seite 448) zu einem ähnlichen abnormen Resultate kam. Heckex fand unter 432 Friuchten älterer Erstgebärenden 247 Knaben und 185 Mädchen. Dies ergiebt eine Proportion von 133: 100. Hecker knüpft an dieses Resultat die Worte: Während der Letztere (Ahlfeld) mit Recht die Sache noch unentschieden liess, so wird sich wohl jetzt mit ziemlicher Sicherheit behaupten lassen, dass dieses Knabenübergewicht nicht ein zufälliges sein kann, sondern als eine den alten Erstgebärenden zu vindicirende Eigenthümlichkeit betrachtet werden muss, die vielleicht für die Länge über die Entstehung des Geschlechtes nicht ganz ohne Bedeutung ist.

Leider ist bisher noch keine Publication erfolgt, mir wenigstens ist keine bekannt geworden, die durch grössere Zahlen das interessante Resultat bestätigt oder verworfen hat. Ich habe deshalb Herrn Dr. Schramm veranlasst, obiges Thema in seiner Inaugural. dissertation zu behandeln. Da die Dissertation als solche nicht gedruckt, und nur Theile derselben in sehr veränderter Weise in den Mittheilungen des statistischen Bureaus der Stadt Leipzig, 10. Heft, 1876, erscheinen sind, so hat Herr Dr. Schramm mir gestattet, die Tabellen, welche zur Klärung der erwähnten Frage von Wichtigkeit sind, in diesem Archive mitzutheilen.

Schramm benutzte als Material die Protokolle von 5218 Geburten in der Leipziger Entbildungsanstalt, 2448 Geburten der Polikhinik und 8680 Geburten, die dem statistischen Bureau der Stadt Leipzig angezeigt waren. Unter diesen 16346 Geburten fanden sich 1038 von älteren Erstgebärenden, die 28 Jahre und darïber alt 
waren. Davon gehörten 278 Protokolle der Entbindungsanstalt, 201 der Poliklinik, und 559 dem statistischen Bureau. Den 1038 Frauen wurden 1055 Kinder geboren.

In der Klinik sind registrirt 156 Knaben, 123 Mädchen $=127: 100$

, , Poliklinik sind regi-

$$
\text { strirt .. . . . } 117 \quad, \quad 91 \quad, \quad=128,5: 100 .
$$

, dem statistischen Bureau

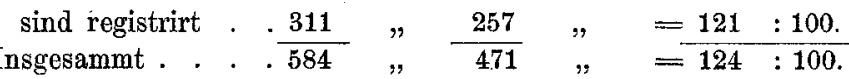

Obgleich der Knabenüberschuss nicht so gross ist, wie Hecker und ich ihn bei älteren Erstgebärenden gefunden habe, so ist er doch immer noch gravirend genug, um die Thatsache zu bestätigen, dass Frauen, die im vorgerücktem Alter zum ersten Male schwanger werden, unverhältnissmässig mehr Knaben als Mädchen gebären.

Auch die Differenz zwischen den Hauptresultaten von Schramm, Hecker und dem meinigen lässt sich begründen, wenn wir eine weitere Tabelle aus Schramm's Arbeit zu Hülfe nehmen. Es ergiebt sich nämlich, dass, je älter eine Erstgebärende ist, desto grösser ist der Knabenüberschuss, der geboren wird.

Von 271 Frauen, im Alter von $28 \mathrm{~J}$. wurden $143 \mathrm{Kn} .130 \mathrm{M}$. geboren $=110: 100$.

$$
\begin{aligned}
& \text { " } 168 " \text { " " } " 29, \quad, 93,79, \quad "=118: 100
\end{aligned}
$$

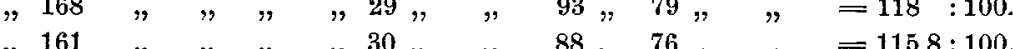

$$
\begin{aligned}
& " 161 \quad " \quad " \quad \text { " } \quad 30, \quad \text { " } \quad 88,76, \quad " \quad=115,8: 100 \text {. }
\end{aligned}
$$

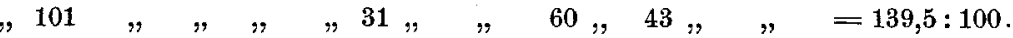

$$
\begin{aligned}
& \text { " } 80 \quad " \quad, \quad, \quad, 32, \quad, \quad 42, \quad 38, \quad, \quad=110,5: 100 \text {. } \\
& \text { " } 64 \quad " \quad " \quad, 33 \% \quad \text { " } \quad 37,29, \quad " \quad=127,6: 100 \text {. } \\
& \text { " } 58 \text { " " " } " 34 ", \quad 35,23, \quad "=152: 100 \text {. } \\
& \text { " } 39 \quad " \quad " \quad, 35, \quad, \quad 29, \quad 11 \% \quad " \quad=265,4: 100 \text {. } \\
& \text { " } 29 \quad " \quad, \quad, 36, \quad, \quad 19, \quad 10 " \quad, \quad=190 \quad 100 \text {. } \\
& \text { " } 23 \quad " \quad \text { " " } 37, \quad \text { " } 13,13, \quad " \\
& \text { " } 16 \quad " \quad \text { " }, 38, \quad \text { " } \quad 13, \quad 3 \% \quad \text { " } \\
& \text { " } 9 \quad " \quad, \quad, \quad, 39, \quad, \quad 3 \% \quad 6 \%, \\
& \text { " } 3 \quad \text { " } \quad, \quad, 40, \quad, \quad 1, \quad 2, \quad \text {, } \\
& \text { " } 9 \quad " \quad \text { " } \quad \text { " } 41, \quad \text { " } 7 \text { " } 2 \text { " }
\end{aligned}
$$

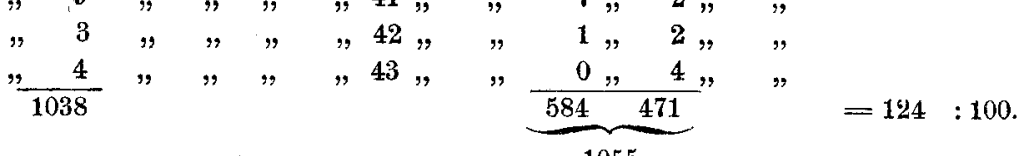

Wenn auch die Procentzahlen zu Gunsten des Knabenüberschusses nicht in ganz regelmässiger Reihenfolge zunehmen, so unterliegt es doch keinem $Z_{w}$ eifel, dass proportional dem Alter der Frauen auch der Knabenüberschuss zunimmt. Wenn für diese Tabelle sehr grosse Zahlen benutzt würden, so würde, vielleicht bis zu einer gewissen Grenze, möglichen Falls aber auch bis zum höchsten befruchtungsfähigen Alter hinauf, ein gleichmässiges Steigen sich documentiren. 
Diese Tabelle erklärt auch die Differenzen in den Hauptresultaten der drei genannten Autoren. Schramm hat ältere Erstgebärende vom 28. Jahre an, Hecker vom 30, ich vom 32. Jahre an ausgewählt. Dem entsprechend fand Schramm 124:100, Hecker $133: 100$, ich $137: 100$.

Berechnet man aus Schxamm's Material das Verhältniss von Knaben und Mädchen bei den Frauen, die 30 Jahre und darüber alt sind, so erbält man die Zahlen 132,8: 100, also fast genau dieselben, die $\mathrm{Hecker}$ fand. Berechnet man das Verhältniss bei Frauen von 32 Jahren und dariber, so findet man 139,8:100, also ein um ein weniges grösseres Verhältniss, als ich es gefunden habe.

Diese beiden Zahlen lassen ihrer Aehnlichkeit halber annehmen, dass ein ganz bestimmtes, sich wenig verändeindes Gesetz die Anzahl der Knaben zu den Mädchen bei den älteren Erstgebärenden regelt.

Es wäre nun sehr interessant zu wissen, ob diese regelmässige Zunahme des Knabenüberschusses auch weiter zurück in annähernd gleichen Verhältnissen vorhanden ist. Es wäre daher eine' sehr dankenswerthe Arbeit, wenn aus den Journalen einer oder mehrerer grösserer Anstalten nicht nur die in S $\mathrm{ehra} \mathrm{mm}$ 's Arbeit behandelten Fragen controlirt würden, sondern auch die Procentverhältnisse der Früchte bei 26jährigen, 24jährigen, 22jährigen, 20jährigen Erstgebärenden mit Einschluss aller später folgenden Jahre erörtert würden. Es ist ungefähr zu erwarten, dass entsprechend der absteigenden Progression 139:133:124 für die Frauen älter als 32, 30, 28 Jahre, für die Frauen älter als 26, 24, 22, 20 Jahre endlich das normale Verhältniss von $106: 100$ resultiren würde.

Ich habe im 2. Hefte dieses Bandes auf eine Schlussfolgerung von Schultze aufmerksam gemacht, der gemäss man bei einer Frau das Vorhandensein von männlichen und weiblichen Eiern annehmen muss. Die Entwickelung des Geschlechtes würde danach ganz unabhängig vom männlichen Sperma vor sich gehen. Auch die eben gewonnenen Thatsachen bestätigen wiederum, dass im Organismus der Frau die Hauptgründe für das spätere Geschlecht ihrer Kinder liegen.

Das Hofacker-Sadler'sche Gesetz würde, wenn sich unsere Annahme bewahrheitete, in der That einen schweren Stoss zu erleiden haben. Ehe ich die Arbeiten über die Geburten älterer Erstgeschwängerten gemacht hatte, controlirte ich seiner Zeit das Hofacker-Sadler'sche Gesetz durch ein Material, welches mir vom Leipziger Polizeiamte, bei dem die Geburtsanmeldungen zu erfolgen hatten, in liberaler Weise zar Verfügung gestellt wurde.

Von dem Gedanken ausgehend, dass man sich ein Urtheil über den Werth des Hofacker-Sadler'schen Gesetzes am sichersten dann bilden könne, wenn man, die im gewöhnlichen Leben bestehenden Altersdifferenzen zwischen Eheleuten bei Seite lassend, nur Fälle bei der Berechnung benutzt, in denen eine grössere Altersdiffereaz besteht, sind dieser Arbeit zu Grunde gelegt die Notizen 
von 500 Familien, in denen Mann und Frau mindestens $10 \mathrm{Jahre}$ im Alter auseinander stehen.

Man kann sich denken, dass, wenn die Frau 10 Jahre älter ist als der Mann, von reichem Kindersegen nicht viel mehr die Rede sein wird. Ja, in der grösseren Anzahl der Fälle, wurden gar keine Kinder in solchen Ehen geboren. So blieb die Zahl der Kinder eine zu geringe, um Rückschlüsse daraus ziehen zu können.

In 439 Ehen waren die Männer älter als die Frauen, in 61 Ehen die Frauen älter als die Männer.

In letzteren 61 Ehen waren nur 22 fruchtbar und zwar wurden geboren bei einer Differenz im Alter von

$$
\begin{aligned}
& 10 \text { Jahren in } 7 \text { Ehen } 9 \text { Knaben } 7 \text { Mädchen, }
\end{aligned}
$$

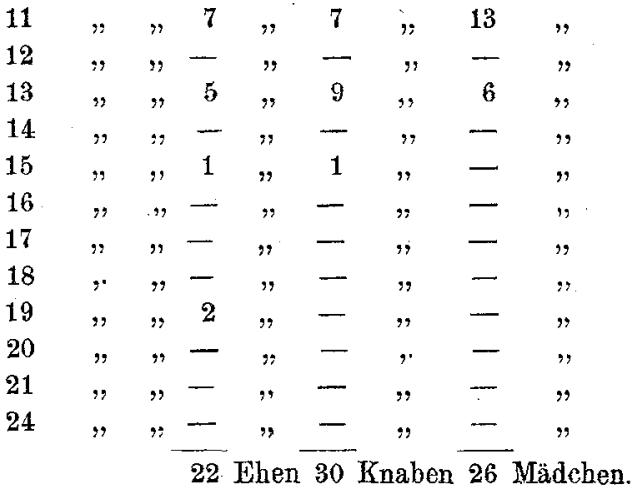

Die Resultate aus den Ehen, in welchen die Männer älter waren, fasse ich am Besten in Tabellenform zusammen.

In 439 Ehen wurden 1376 Kinder geboren.

\begin{tabular}{|c|c|c|c|c|c|c|}
\hline \multirow{2}{*}{\multicolumn{2}{|c|}{ Mann älter }} & \multicolumn{2}{|c|}{ Ehen } & \multicolumn{2}{|c|}{ Geboren wurden } & \multirow[b]{2}{*}{ Summa } \\
\hline & & unfruchtb. & fruchtb. & Knaben & Mädchen & \\
\hline 10 & Jahre & 8 & 88 & 168 & 165 & 333 \\
\hline 11 & $"$ & 4 & 55 & 90 & 117 & 207 \\
\hline 12 & $\eta$ & 7 & 58 & 103 & 84 & 187 \\
\hline 13 & $"$ & 3 & 38 & 73 & 65 & 138 \\
\hline 14 & $"$ & 5 & 34 & 57 & 59 & 116 \\
\hline 15 & $"$ & 3 & 24 & 30 & 38 & 68 \\
\hline 16 & $n$ & 3 & 23 & 43 & 45 & 88 \\
\hline 17 & $"$ & 1 & 15 & 30 & 24 & 54 \\
\hline 18 & $\eta$ & 2 & 10 & 11 & 14 & 25 \\
\hline 19 & $"$ & 4 & 18 & 35 & 28 & 63 \\
\hline 20 & $"$ & - & 8 & 11 & 14 & 25 \\
\hline 21 & $n$ & 1 & 1 & 1 & 3 & 4 \\
\hline 22 & $"$ & 1 & 5 & 3 & 8 & 11 \\
\hline 23 & $"$ & 1 & 6 & 13 & 11 & 24 \\
\hline 24 & $"$ & - & - & - & - & - \\
\hline 25 & " & - & 2 & 2 & 2 & 4 \\
\hline 26 & $"$ & - & 1 & 1 & 4 & 5 \\
\hline & & & & & $30^{*}$ & \\
\hline
\end{tabular}




\begin{tabular}{|c|c|}
\hline \multicolumn{2}{|c|}{ Mann älter } \\
\hline & Jahre \\
\hline & $n$ \\
\hline & $"$ \\
\hline & , \\
\hline & ", \\
\hline & $"$ \\
\hline & $\eta$ \\
\hline & $"$ \\
\hline
\end{tabular}

10 bis 15 Jahre
Ehen unfruchtb. fruchtb.
Geboren wurden Knaben Mädchen

Summa

\begin{tabular}{rr}
1 & 3 \\
- & - \\
4 & 3 \\
2 & 3 \\
- & - \\
2 & 1 \\
2 & 2 \\
- & 1 \\
\hline 682 & 694
\end{tabular}
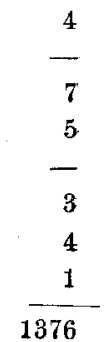

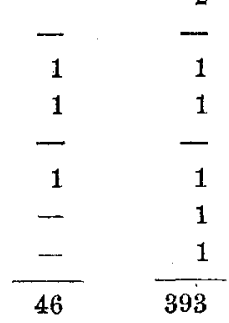

Das Endresultat giebt eine grössere Anzahl Mädchen als Knaben. Statt des normalen Verhältnisses von 106 : 100 erhalten wir hier ein umgekehrtes 98,2: 100 .

Es' könnte mir entgegnet werden, dass durch die hohen Altersdiffereuzen von 20 Jahren und darüber Momente mitwirkten, die uns noch unbekannt, v́ielleicht die Resultate des Hofacker-Sadler'schen Gesetzes trüben könnten. Lassen wir deshalb die höchsten Differenzen im Alter bei Seite und rechnen nur mit den Ehen; in dener der Mann 10-15 Jahre älter ist als die Frau. Wir erhalten:

\begin{tabular}{ccccc}
\multicolumn{2}{c}{ Ehen } & \multicolumn{2}{c}{ Geboren wurden } & Summa \\
unfruchtbare & fruchtbare & Knaben & Mädchen & \\
30 & 297 & 521 & 528 & 1049
\end{tabular}

Das Gesammtverhältniss ist 98,6:100, ähnelt also dem aus. sämmtlichen Ehen gefundenen ziemlich genau.

Auch Schramm hat in seiner Arbeit eine Tabelle geliefert, in welcher er die Ehen zusammengestellt hat, in denen der Mann fünf und mehr Jahre älter als die Frau war. Fall war:

In seinem Materiale fanden sich 126 Ehen, in denen dies der

\begin{tabular}{crrrrrrr} 
Mann älter & \multicolumn{3}{c}{ Ehen } & \multicolumn{2}{c}{ Geboren wurden } & Mann älter & \multicolumn{3}{c}{ Geboren wurden } \\
Jahre & Knn. & M. & Jahre & Ehen & Kn. & M. \\
5 & 44 & 71 & 85 & 14 & 2 & 3 & 4 \\
6 & 5 & 6 & 8 & 15 & 9 & 16 & 6 \\
7 & 10 & 19 & 17 & 16 & 1 & 3 & 0 \\
8 & 10 & 20 & 26 & 17 & 2 & 2 & 2 \\
9 & 4 & 8 & 6 & 18 & 1 & 1 & 1 \\
10 & 16 & 21 & 25 & 21 & 1 & 0 & 1 \\
11 & 10 & 22 & 20 & 23 & 1 & 0 & 1 \\
12 & 6 & 8 & 8 & 5-23 J. & $\mathbf{1 2 6}$ & $\mathbf{2 0 7}$ & $\mathbf{2 1 3}$ \\
13 & 4 & 7 & 3 & & & &
\end{tabular}

Das Gesammtverhältniss 97,18:100 stimmt mit dem meinigen auffallend. Die Wahrscheinlichkeit wächst somit, dass nicht das gegenseitige Alter der Eltern; sondern nur das Alter der Mutter das. Ausschlag gebende Moment bei der Entwickelung des Geschlechtes ist. 
Weitere Schlüsse zu ziehen, die sehr nahe liegen, will ich zur Zeit unterlassen, bis erst durch grössere statistische Zusammenstellungen die von uns gefundenen Resultate als unumstössliche dastehen.

Auf meinen Rath hat Herr Dr. Schramm auch das Verhältniss der Knaben zu den Mädchen in der jüdischen Gemeinde mit in den Bereich seiner Arbeit gezogen. Die Zahlen, welehe ihn in Leipzig zu Gebote standen, sind leider sehr klein." Doch hat er verschiedene Notizen aus der Literatur über diesen Punkt zusammengetragen, die sehr lesenswerth sind. Ich verweise in dieser Hinsicht auf das Original.

\section{Zur Frage ron der pathologischen Antefiexion der Gebär- matter.}

Yon

B. S. Schultze.

Die combinirte Untersuchung lehrt uns nicht allein, dass der Uterus durch die palpirende Hand leicht bewegt werden kann, sondern auch, dass er olne unser Zuthun dureh die wechselnde Füllung der Nachbarorgane täglich Bewegungen macht. Während er bei mässig stark gefüllter Blase mit seiner Axe etwa in der Axe des Beckeneinganges liegt, bei stärkerer Füllung weiter rückwärts ge drängt wird, tritt er bei Entleerung der Blase mit dem Fundus nach vorn in antevertirte, gleichzeitig mehr oder minder stark an teflectirte Stellung. Die Lageveränderung, welche der Uterus durch Entleerung der Blase erleidet, ist also gleichzeitig von einer Aenderung der Gestalt normaler Weise begleitet.

Wenn wir von der normalen Lage des Uterus reden, können wir deshalb jetzt nicht mehr wie früher eine bestimmte constante Lage im Sinne haben, sondern zur "normalen Lage" des Uterus gehört unveräusserlich nicht nur ein gewisser Grad von Beweglichkeit, sondern auch das factische Stattfinden gewisser Bewegungen desselben.

Während wir früher das Nachvornhiegen des Fundus uteri (Anteversion und Anteflexion) an sich und jedes Mal für pathologisch hielten, kennen wir jetzt auch eine normale Anteflexion und wissen, dass jeder normale Uterus mehrmals täglich in derselben sich befindet.

Dem entsprechend ändert sich nothwendig die Definition der pathologischen Anteflexion. Nahe liegend wäre es gewesen, den Grad des Knickungswinkels als Kriterium der pathologischen 